\title{
Dual beam delivery system serving two interaction regions for the Compact Linear Collider
}

\author{
Vera Cilento®, ${ }^{*} \dagger$ Rogelio Tomás, and Benoit Cure \\ CERN, European Organization for Nuclear Research, Geneva CH-1211, Switzerland \\ Angeles Faus-Golfe \\ Laboratoire de Physique des 2 Infinies, Irene Joliot-Curie Laboratory IJCLab, CNRS/IN2P3, \\ Universit'e Paris-Saclay, Orsay 91898, France \\ Barbara Dalena \\ CEA, Irfu, DACM, Université Paris-Saclay, F-91191, Gif-sur-Yvette, France \\ Yngve Levinsen 1 \\ European Spallation Source (ESS), 22484 Lund, Sweden
}

(Received 18 May 2021; accepted 12 July 2021; published 29 July 2021)

\begin{abstract}
The Compact Linear Collider (CLIC) could provide $e^{+} e^{-}$collisions in two detectors simultaneously possibly at a bunch train frequency in the linac twice the baseline design value. In this paper, a novel dual beam delivery system (BDS) design is presented in order to serve two interaction regions (IR1 and IR2) including optics designs and the evaluation of luminosity performance with synchrotron radiation (SR) and solenoid effects for both energy stages of CLIC, $380 \mathrm{GeV}$ and $3 \mathrm{TeV}$. IR2 features a larger crossing angle than the current baseline. The luminosity performance of the novel CLIC scheme was evaluated by comparing the different BDS designs with and without the detector solenoid field effects. It has to be highlighted that the impact of the detector solenoid on luminosity had not been evaluated for the current CLIC baseline, which amounts to a loss of about $4 \%$ that corresponds to the same value of the old baseline design. At $380 \mathrm{GeV}$ the novel dual BDS design features same luminosities than the current baseline. However at $3 \mathrm{TeV}$ the luminosity performance is reduced by $2 \%$ from the baseline design for the IR 1 and by $33 \%$ for the IR2. The dual CLIC BDS design provides adequate luminosities to two detectors and proves to be a viable candidate for future linear collider projects.
\end{abstract}

DOI: 10.1103/PhysRevAccelBeams.24.071001

\section{INTRODUCTION}

A lepton linear collider is considered as one of the potential candidates to continue the high precision particle physics research after the discovery of the Higgs boson at the Large Hadron Collider (LHC) and further study in its luminosity upgrade, HL-LHC [1]. In particular, two machines have been proposed for the linear collider accelerator physics scenario: the International Linear Collider (ILC) with a center of mass (c.o.m.) energy of

\footnotetext{
*vera.cilento@cern.ch

†Also at Laboratoire de Physique des 2 Infinies, Irene JoliotCurie Laboratory IJCLab, CNRS/IN2P3, Université Paris-Saclay, Orsay, France.

Published by the American Physical Society under the terms of the Creative Commons Attribution 4.0 International license. Further distribution of this work must maintain attribution to the author(s) and the published article's title, journal citation, and DOI.
}

$250 \mathrm{GeV}$ with a proposed upgrade to $1 \mathrm{TeV}[2,3]$ and CLIC with a c.o.m. energy of $380 \mathrm{GeV}$ with a possible upgrade to $3 \mathrm{TeV}[4,5]$.

This research is focused on the CLIC machine and more specifically on the CLIC beam delivery system (BDS) [6] that transports the $e^{+}$and $e^{-}$beams from the exit of the linacs to the interaction region (IR) by performing the critical functions required to meet the CLIC luminosity goals of $1.5 \times 10^{34} \mathrm{~cm}^{-2} \mathrm{~s}^{-1}$ for CLIC $380 \mathrm{GeV}$ and $5.9 \times 10^{34} \mathrm{~cm}^{-2} \mathrm{~s}^{-1}$ for CLIC $3 \mathrm{TeV}$ [7].

CLIC current baseline design includes only one detector and one BDS. In $[7,8]$ the baseline designs for both CLIC $380 \mathrm{GeV}$ and $3 \mathrm{TeV}$ were optimized including moving the final quadrupole (QD0) outside the detector. The distance between QD0 and the interaction point (IP) was increased to $\mathrm{L}^{*}=6 \mathrm{~m}$.

The previous versions with $\mathrm{L}^{*}=4.3 \mathrm{~m}$ for CLIC $380 \mathrm{GeV}$ and $\mathrm{L}^{*}=3.5 \mathrm{~m}$ for CLIC $3 \mathrm{TeV}$ featured an antisolenoid to cancel the magnetic field inside QD0, while the $\mathrm{L}^{*}=6 \mathrm{~m}$ does not. The $\mathrm{L}^{*}=6 \mathrm{~m}$ design is considered 
TABLE I. CLIC BDS design parameters.

\begin{tabular}{lcccc}
\hline \hline CLIC & $380 \mathrm{GeV}$ & \multicolumn{2}{c}{$3 \mathrm{TeV}$} \\
\hline $\mathrm{L}^{*}[\mathrm{~m}]$ & 4.3 & 6 & 3.5 & 6 \\
BDS length [m] & 1728 & 1949 & 2795 & 3117 \\
Normalised emittance $\gamma \epsilon_{x}[\mathrm{~nm}]$ & 950 & 950 & 660 & 660 \\
Normalised emittance $\gamma \epsilon_{y}[\mathrm{~nm}]$ & 30 & 30 & 20 & 20 \\
Beta function (IP) $\beta_{x}^{*}[\mathrm{~mm}]$ & 8 & 8 & 7 & 7 \\
Beta function (IP) $\beta_{y}^{*}[\mathrm{~mm}]$ & 0.1 & 0.1 & 0.068 & 0.12 \\
IP beam size $\sigma_{x}^{*}[\mathrm{~nm}]$ & 144 & 144 & 40 & 40 \\
IP beam size $\sigma_{y}^{*}[\mathrm{~nm}]$ & 2.9 & 2.9 & 0.7 & 0.9 \\
Bunch length $\sigma_{z}[\mu \mathrm{m}]$ & 70 & 70 & 44 & 44 \\
rms energy spread $\delta_{p}[\%]$ & 0.3 & 0.3 & 0.3 & 0.3 \\
Bunch population $N_{e}\left[10^{9}\right]$ & 5.2 & 5.2 & 3.72 & 3.72 \\
Number of bunches $n_{b}$ & 352 & 352 & 312 & 312 \\
Repetition rate $f_{\text {rep }}[\mathrm{Hz}]$ & 50 & 50 & 50 & 50 \\
Crossing angle $[\mathrm{mrad}]$ & 18.3 & 16.5 & 20 & 20 \\
Luminosity $\mathcal{L}_{\mathrm{TOT}}\left[10^{34} \mathrm{~cm}^{-2} \mathrm{~s}^{-1}\right]$ & 1.5 & 1.5 & 5.9 & 5.9 \\
\hline \hline
\end{tabular}

as the starting point for the development of the model for the dual CLIC BDS. Table I summarizes the design parameters of CLIC baseline designs, for both energies options and both L* options. Figure 1 shows the CLIC BDS layout of the different baseline designs, where c.a. is the crossing angle. Dual BDS designs were already considered for the next linear collider (NLC) [9], where luminosity perfomance for the two detectors was within $30 \%$ up to the energy of $1.3 \mathrm{TeV}$. In this paper we present a new design with comparable luminosity imbalance up to an energy of $3 \mathrm{TeV}$.

ILC [2] has a single IP and two detectors can take data in different periods of time following a push-pull arrangement. A dual BDS preliminary layout has been proposed
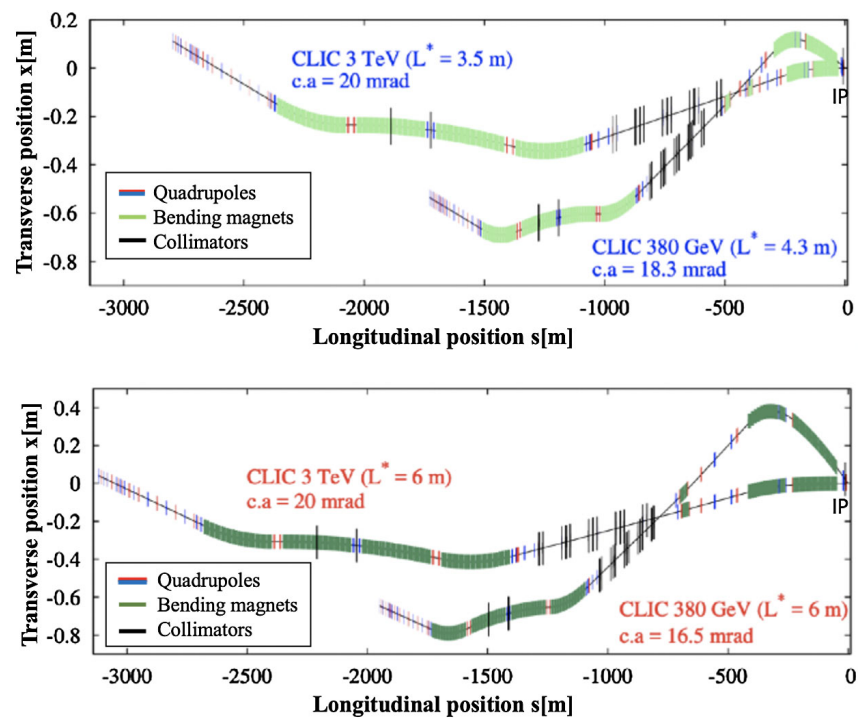

FIG. 1. BDS Layout of CLIC baseline designs. Top: option with $\mathrm{L}^{*}=4.3 \mathrm{~m}$ for CLIC $380 \mathrm{GeV}$ and $\mathrm{L}^{*}=3.5 \mathrm{~m}$ for CLIC $3 \mathrm{TeV}$ [6]. Bottom: option with the long $\mathrm{L}^{*}\left(\mathrm{~L}^{*}=6 \mathrm{~m}\right.$ for both energy cases). The IP is at $0 \mathrm{~m}$ [8]. also for ILC allowing two distinct interaction regions with crossing angles of $2 \mathrm{mrad}$ and $20 \mathrm{mrad}$ respectively [10,11].

The objective of this work is to present a novel dual BDS system scheme, feasible in terms of luminosity, in order to allocate two detectors and to make CLIC more competitive with other future circular collider projects.

One of the main design drivers is the luminosity loss due to emittance increase and related widening of the beam sizes caused by the emission of synchrotron radiation (SR) as generated in the bending magnet section in the first added part of the BDS, called Diagnostics section (DS), to separate the 2 IRs. This effect is much more significant for the CLIC $3 \mathrm{TeV}$ case, since the contribution to the IP beam size scales with the fifth power of energy [12]:

$$
\sigma_{\text {bend }}^{2}=C_{2} \int_{0}^{s_{p}} \frac{E^{5}}{\rho^{3}} R_{16}\left(s, s_{p}\right)^{2} d s,
$$

where $C_{2}=\frac{55}{24 \sqrt{3}} \frac{r_{e} h c}{\left(m c^{2}\right)^{6}}=4.13 \times 10^{-11} \mathrm{~m}^{2} \mathrm{GeV}^{-5}$ is a constant coming from the emission rate integration derived in [13], $\mathrm{E}$ is the beam energy, $\rho$ is the bending radius and $R_{16}$ is the transfer matrix element between $s$ and $s_{p}$. To mitigate luminosity loss from this contribution $\rho$ needs to be as large as possible, which determines the length of the BDS.

Another important design parameter is the crossing angle at IP. In fact, the SR in the final quadrupole and the solenoid increases the vertical spot size at the IP. In [14] it was calculated that this growth was acceptable for $\theta_{C} \leq 20 \mathrm{mrad}$ in a $4 \mathrm{~T}$ solenoid field for CLIC $3 \mathrm{TeV}$, leading to the current baseline value of $20 \mathrm{mrad}$. In the proposed dual detector configuration the new IR will necessarily feature a larger crossing angle and this effect will be evaluated.

An interesting consequence of adding a new IR with larger crossing angle is its compatibility with gammagamma collisions [15]. A crossing angle of about $25 \mathrm{mrad}$ is considered optimal for this type of collisions [16].

The impact of the detector solenoid on CLIC luminosity performance was in part studied in [17] for the $\mathrm{L}^{*}=3.5 \mathrm{~m}$ design for CLIC $3 \mathrm{TeV}$. The machine detector interface (MDI) design taken into account in that study was the SiD experiment $[18,19]$ shown in Fig. 2. This layout places the final quadrupole QD0 inside the experiment and imposes the integration of a preinsulator system and an active insulation to mitigate vibrations of QD0 inside the detector to the $0.1 \mathrm{~nm}$ level. Additionally, due to the presence of a strong magnetic field, higher radiation, and lack of space and access inside the detector, some critical components require longer interventions, leading to a loss of integrated luminosity. The QD0 being installed inside the detector takes away a significant fraction of the acceptance in the forward region. This is partially due to the need of shielding QD0 with an antisolenoid in order to reduce the interplay between the solenoid and the QD0 fields, which would otherwise limit quadrupole field and reduce luminosity [7]. 

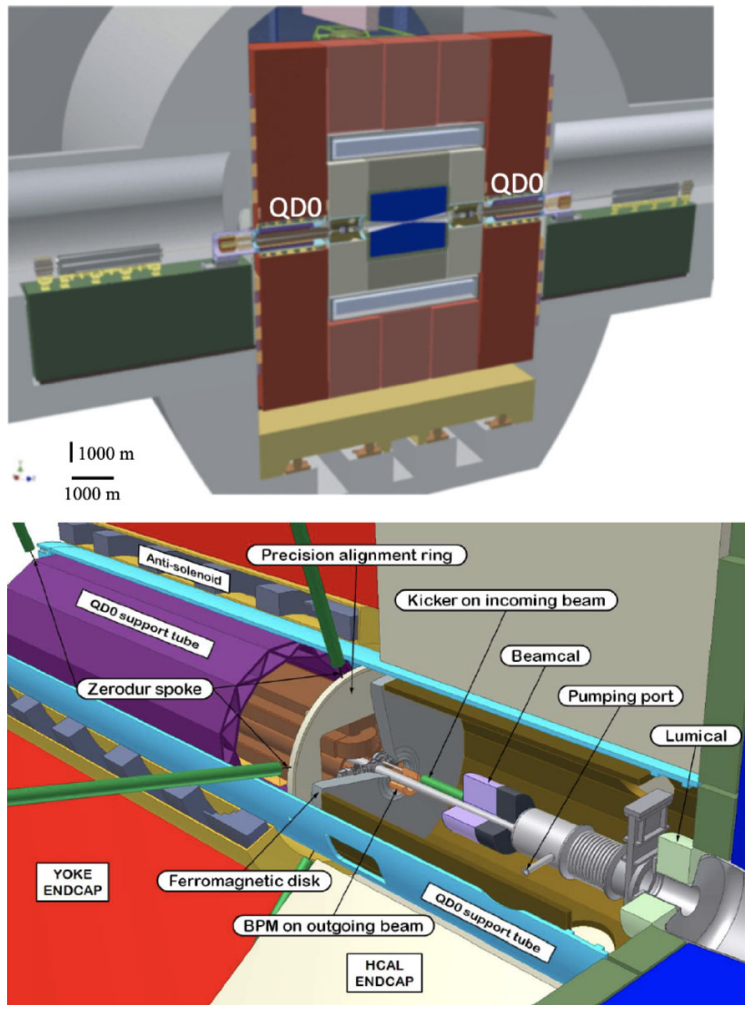

FIG. 2. Top: Vertical cut through the SiD experiment for CLIC $3 \mathrm{TeV}$. QD0 is located inside the detector and partially supported by the preinsulator (green block) in the tunnel. The antisolenoid is present for shielding QD0 [7]. Bottom: simplified MDI layout view showing a representation of part of the final-focus quadrupole, QD0, integrated into the CLIC SiD detector and shielded by an antisolenoid [18].

The results in this paper take into account the solenoid effect coming from the new design proposed in $[7,8]$ that involved QD0 outside the detector thanks to an increase of $\mathrm{L}^{*}$ up to $6 \mathrm{~m}$ for both CLIC energy options $[7,8]$. This detector is called CLICdet [20] and it is shown in Fig. 3. The preinsulator system is no longer needed, and the access to the detector and QD0 are also simplified. The magnetic shielding of QD0 is not needed, so the antisolenoid is not part of the MDI. The detector cavern size has a half length of $5918 \mathrm{~mm}$ and a half width of $6800 \mathrm{~mm}[6,20]$.

This paper is structured as follows. In Sec. II there is an update of the CLIC $3 \mathrm{TeV}$ performance including the detector solenoid effects, explaining in detail the tracking procedure for the evaluation of the detector solenoid effects and presenting the new results. The CLIC $380 \mathrm{GeV}$ case was not studied since the solenoid effects can be considered negligible for this case. In Sec. III a detailed description of the design of the dual BDS is presented, with at first a focus on CLIC $380 \mathrm{GeV}$ then, the main features and challenges of the energy upgrade are taken into consideration. Section IV focuses on the performances: they are presented in terms of beam size and achievable luminosity with the new dual BDS configuration considering only the SR
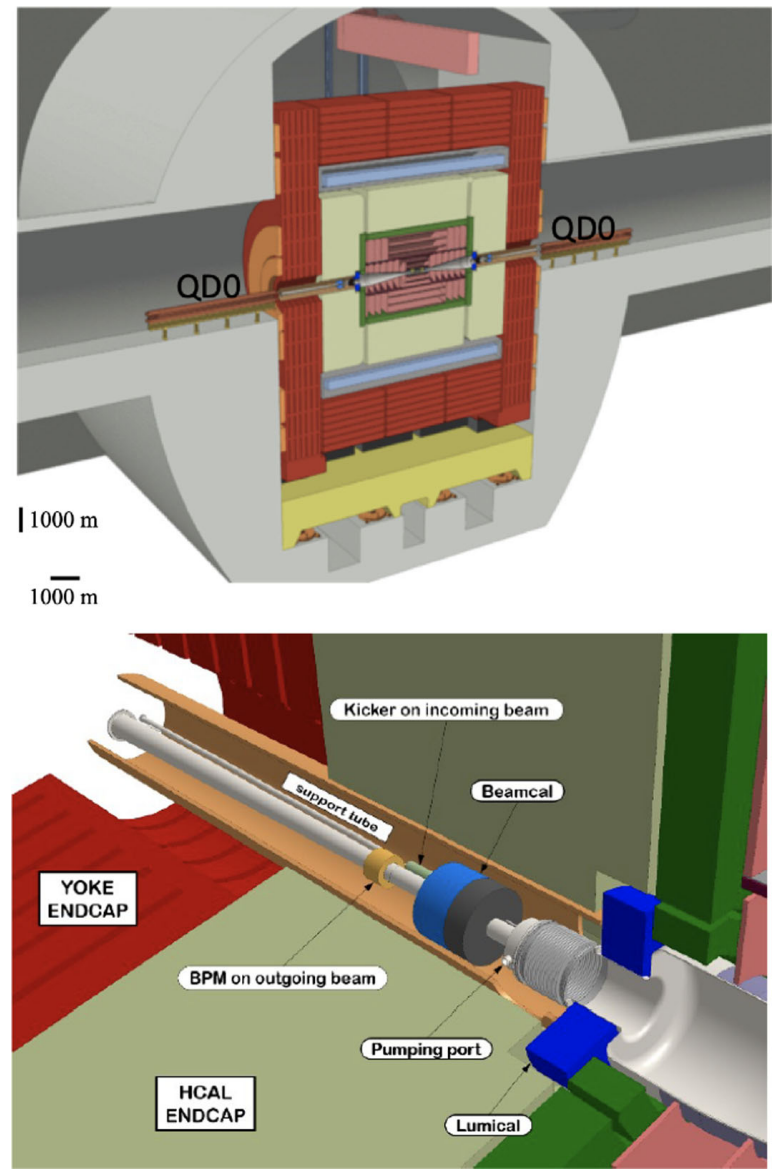

FIG. 3. Top: Vertical cut through the new detector model CLICdet allowing QD0 to be located outside of the experiment for CLIC $3 \mathrm{TeV}$. No preinsulator or QD0 shielding with antisolenoid are needed as opposed to the short $\mathrm{L}^{*}$ design in Fig. 2 [7]. Bottom: Forward region of the CLICdet experiment [21].

effects in the first part, while in the second part the luminosity is calculated considering also the detector solenoid effects. The last section discusses the results and presents the conclusions.

\section{UPDATE OF CLIC PERFORMANCE INCLUDING THE DETECTOR SOLENOID EFFECTS}

In this section the performance of the CLIC $3 \mathrm{TeV}$ design with $\mathrm{L}^{*}=6 \mathrm{~m}[7,8]$ is evaluated including the detector solenoid field for the first time.

\section{A. Tracking procedure to assess the detector solenoid effects}

Particle tracking simulations are done with PLACET [22] and GUINEA-PIG [23]. The code to asses the solenoid effects has been developed in [17], where the procedure to include the solenoid field map is also described. The tracking including the solenoid field is done using a new 
TABLE II. Beam size and luminosity simulations evaluated with the direct PLACET tracking procedure for the CLIC $3 \mathrm{TeV}$ baseline design with $\mathrm{L}^{*}=6 \mathrm{~m}$ (optics optimized in [8]) and with the forward-backward-forward tracking procedure for the solenoid effects results.

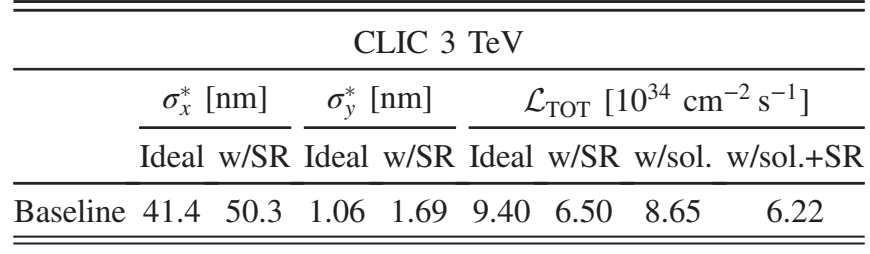

4th order symplectic integrator [17]. The beam is first tracked forward without SR, and without the solenoid field present. This provides the optimal beam distribution at the IP. The ideal IP beam distribution is tracked backwards through the beam line, with the solenoid field turned on but still without SR. The result is a beam distribution with a perfect compensation for the coupling introduced by the solenoid field. Finally, the SR is turned on, and the beam is tracked forward through the solenoid. The estimated luminosity is compared to a normal tracking of the beam without the solenoid field, but including the SR. From now on, this tracking procedure will be called forwardbackward-forward. The results are compared with the direct (classic) tracking procedure to show consistency of results.

\section{B. Results}

The results showing the CLIC baseline (with $\mathrm{L}^{*}=6 \mathrm{~m}$ ) performance including the solenoid are first presented here. The beam size and the luminosity results are presented in Table II. The ideal case does not consider SR and solenoid effects. The two tracking procedures used are the direct PLACET tracking procedure (the classic tracking with PLACET for the results of ideal and with SR) and the forward-backward-forward tracking procedure for the evaluation of the solenoid effects (results of ideal, with solenoid and with solenoid plus SR). The ideal case shows the same performance from the two different tracking procedures, as expected. The luminosity loss from the solenoid field for the current baseline with $\mathrm{L}^{*}=6 \mathrm{~m}$ is about $4 \%$ like for the previous design with $\mathrm{L}^{*}=3.5 \mathrm{~m}$ [17]. The new performance for the current baseline with $\mathrm{L}^{*}=6 \mathrm{~m}$ will be used as the new reference in the following.

\section{DEVELOPMENT OF THE MODEL TO CONSTRUCT THE DUAL BEAM DELIVERY SYSTEM FOR CLIC}

The novel optics design of the dual BDS was performed with MAD-X [24] adding bending sections by extending the DS of the baseline BDS for CLIC $380 \mathrm{GeV}$ and CLIC $3 \mathrm{TeV}$ respectively. The choice of starting separating the
IRs from the DS is mostly to minimize the required dipole field and, hence, the emittance growth from SR. The designs are presented in the following sections for both energy stages.

\section{A. CLIC $380 \mathrm{GeV}$}

The BDS layout is constructed by adding 8 more FODO cells, of a phase advance, $\mu$, of $45^{\circ}$ in the existing DS, with an additional total length of about $300 \mathrm{~m}$. The four BDS systems at either side of the two IPs (BDS1 $e^{ \pm}$and BDS2 $\left.e^{ \pm}\right)$need to have different DS lengths to provide the desired longitudinal and transverse separations at the IP. Figure 4 shows the Twiss functions, $\beta_{x}, \beta_{y}$ and $\eta_{x}$ as function of the longitudinal position along the DS. The first figure on the top shows the existing DS with the FODO cell structure before adding three dipoles, with bending angles $\theta_{1}, \theta_{0}$ and $\theta_{1}$ in agreement with the figure, in order to separate the two BDS.

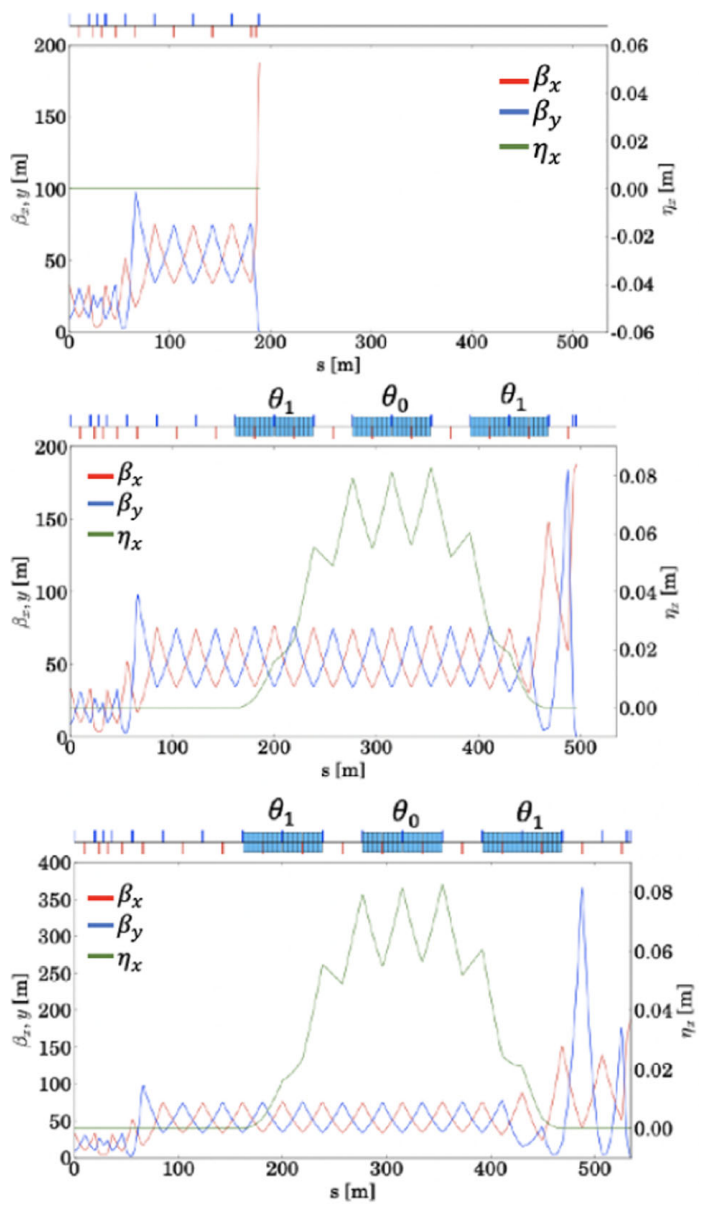

FIG. 4. Top: DS of CLIC $380 \mathrm{GeV}$ for the single IR (baseline design with long $\mathrm{L}^{*}$ ). Middle: new DS for the dual IRs in the case of $\operatorname{BDS} 2 e^{-}$. Bottom: new DS for the dual IRs in the case of the $\operatorname{BDS} 2 e^{+}$. The cases BDS1 $e^{ \pm}$are very similar but with zero dispersion as they have no dipoles. 


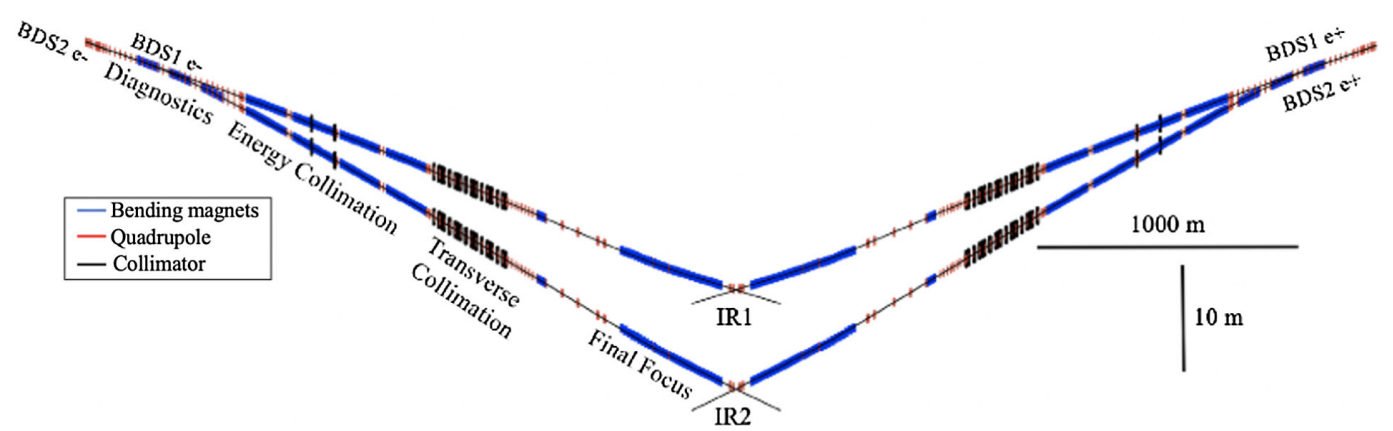

FIG. 5. Layout of the new dual CLIC $380 \mathrm{GeV}$ BDS System for two IRs.

The two figures on the bottom show the optics structure of the DS of the new design for the dual BDS: the middle one, shows the case of the shorter $\operatorname{BDS}\left(\operatorname{BDS} 2 e^{-}\right)$, while the bottom one shows the case of the longer BDS (BDS2 $\left.e^{+}\right)$. On the top of each figure there are the magnets appearing in the DS: in blue the focusing quadrupoles, in red the defocusing ones and in light blue the dipoles. The bending angles are arranged to suppress dispersion at the exit of the DS. The strengths of the dipoles are related as $\theta_{1}=\theta_{0} / \sqrt{2}$ [25]. We define as $\theta$ the total bending angle, $\theta=2 \theta_{1}+\theta_{0}$. The value $\theta=4.83 \mathrm{mrad}$ has been chosen to provide the desired transverse separation of $10 \mathrm{~m}$ between the two detectors to fit the experimental cavern. The Twiss functions at the DS exit have been matched to the design values and then the new DS has been connected to the rest of the BDS in order to get the beam to two different IRs.

The layout of the new dual BDS is shown in Fig. 5 displaying all magnets along the beamlines. Concerning the longitudinal separation of the 2 detectors, it was chosen to be about $40 \mathrm{~m}$ (that corresponds to one FODO cell in the DS), even if it introduces issues with train synchronization, it is necessary in order to minimize the transverse separation space to allocate the two detectors. The two crossing

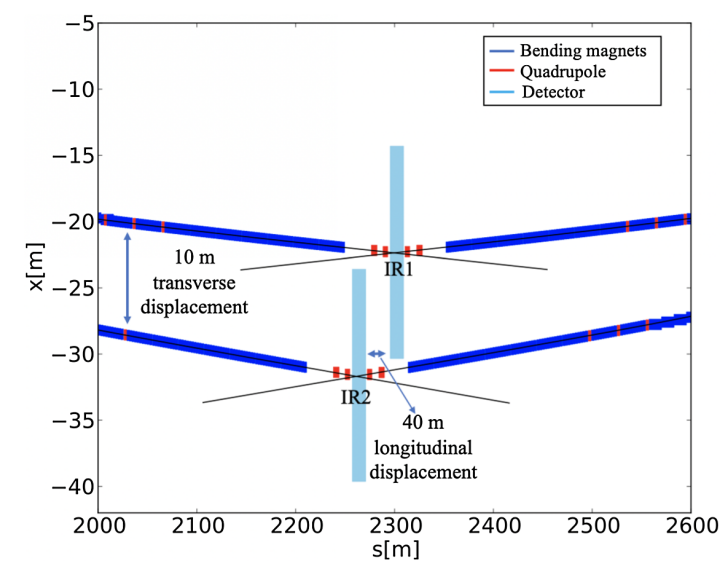

FIG. 6. Zoom at the IRs to have a clear visualization on the longitudinal and transverse separations between the two detectors of about $40 \mathrm{~m}$ and about $10 \mathrm{~m}$, respectively. angles (c.a.) are respectively $16.5 \mathrm{mrad}$ for IR 1 and $26 \mathrm{mrad}$ for IR2 (compatible with gamma-gamma collisions).

A zoom of the IRs is shown in Fig. 6 depicting the different beamlines. IR 1 is longitudinally shifted $40 \mathrm{~m}$ ahead of IR 2 and it is transversely separated by $10 \mathrm{~m}$ from IR2 to allow the necessary cavern sizes to allocate the two detectors without any interference between them.

Table III summarizes the geometrical parameters and the optics functions for CLIC $380 \mathrm{GeV}$.

\section{B. CLIC 3 TeV}

The dual lattice design is also developed for CLIC $3 \mathrm{TeV}$, keeping the compatibility between the 2 energy stages for both IRs. The procedure to make the new beamlines has been the same but in this case the additional length in order to place the dipoles is about $1 \mathrm{~km}$. Figure 7 shows the Twiss functions, $\beta_{x}, \beta_{y}$ and $\eta_{x}$ as function of the longitudinal position along the DS. The first figure on the top shows the existing DS with the FODO cell structure before adding three dipoles in order to separate the two BDS. The two figures on the bottom show the optics structure of the DS of the new design for the dual BDS: the middle one shows the shorter BDS (BDS2 $e^{-}$), while the bottom one shows the longer $\mathrm{BDS}\left(\mathrm{BDS} 2 e^{+}\right)$.

The $\theta$ value is $2.75 \mathrm{mrad}$ to provide exactly the same transverse separation, $10 \mathrm{~m}$, as for the $380 \mathrm{GeV}$ design (the same locations of the IRs) and the crossing angles are for

TABLE III. Summary table of the geometrical parameters and the optics functions for CLIC $380 \mathrm{GeV}$.

\begin{tabular}{lcccc}
\hline \hline \multicolumn{5}{c}{ CLIC 380 GeV } \\
\hline & $\begin{array}{c}\text { BDS1 } e^{+} \\
\text {(short) }\end{array}$ & $\begin{array}{c}\text { BDS1 } e^{-} \\
\text {(long) }\end{array}$ & $\begin{array}{c}\text { BDS2 } e^{-} \\
\text {(short) }\end{array}$ & $\begin{array}{c}\text { BDS2 } e^{+} \\
\text {(long) }\end{array}$ \\
\hline$\theta$ [mrad] & 0 & 0 & 4.83 & 4.83 \\
$L_{\text {dipole }}[\mathrm{m}]$ & 0 & 0 & 218.11 & 218.11 \\
$L_{\text {FODO }}[\mathrm{m}]$ & 38.36 & 38.36 & 38.36 & 38.36 \\
$L_{\text {DS }}[\mathrm{m}]$ & 512.89 & 551.24 & 512.89 & 551.24 \\
$L_{\text {BDS }}[\mathrm{m}]$ & 2255.95 & 2294.3 & 2255.95 & 2294.3 \\
c.a. $[\mathrm{mrad}]$ & 16.5 & 16.5 & 26 & 26 \\
\hline \hline
\end{tabular}




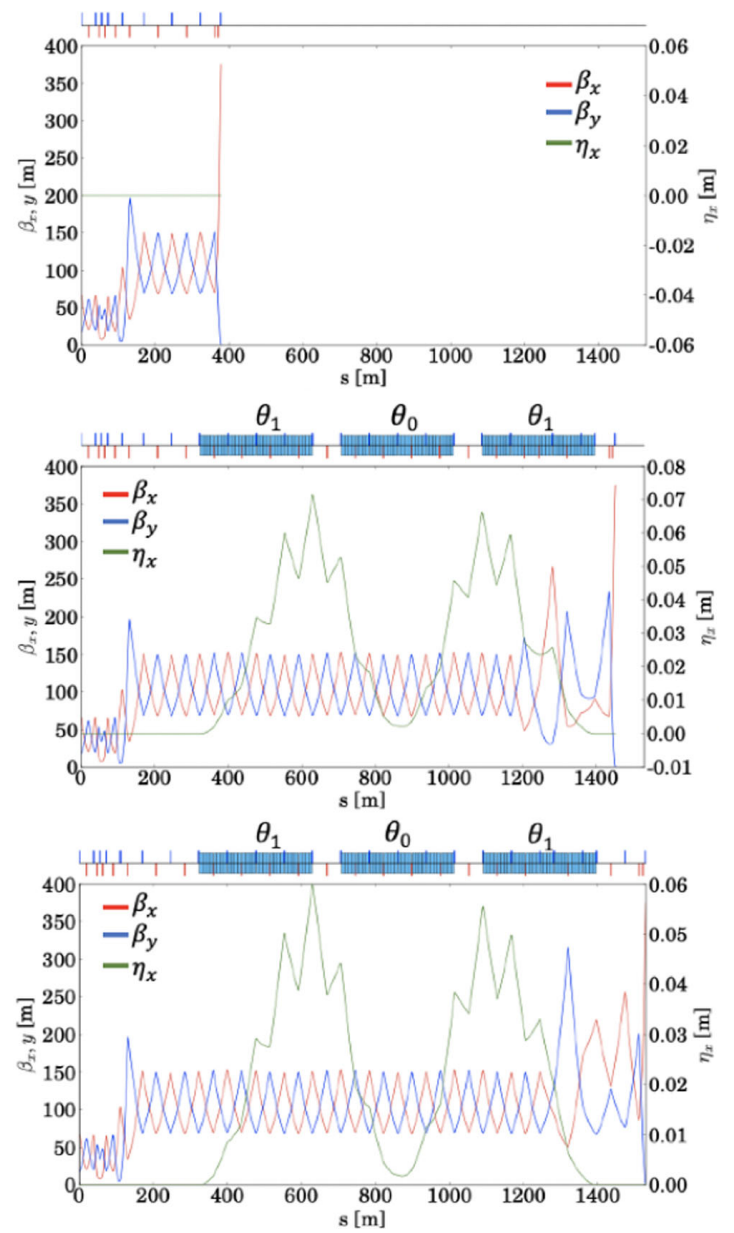

FIG. 7. Top: DS of CLIC $3 \mathrm{TeV}$ for the single IR (baseline design with long $L^{*}$ ). Middle: new DS for the dual IRs in the case of $\operatorname{BDS} 2 e^{-}$. Bottom: new DS for the dual IRs in the case of $\operatorname{BDS} 2 e^{+}$.

IR1 and IR2 respectively $20 \mathrm{mrad}$ and $25.5 \mathrm{mrad}$. The new dual BDS layout design for CLIC $3 \mathrm{TeV}$ option is shown in Fig. 8.

The layout of CLIC at both energy stages and for both BDS, is shown in Fig. 9. The crossing angles (c.a.) are indicated at the different IRs. The layout of CLIC $3 \mathrm{TeV}$ is longer, $4340 \mathrm{~m}$. The $380 \mathrm{GeV}$ layout in contrast, is $2000 \mathrm{~m}$

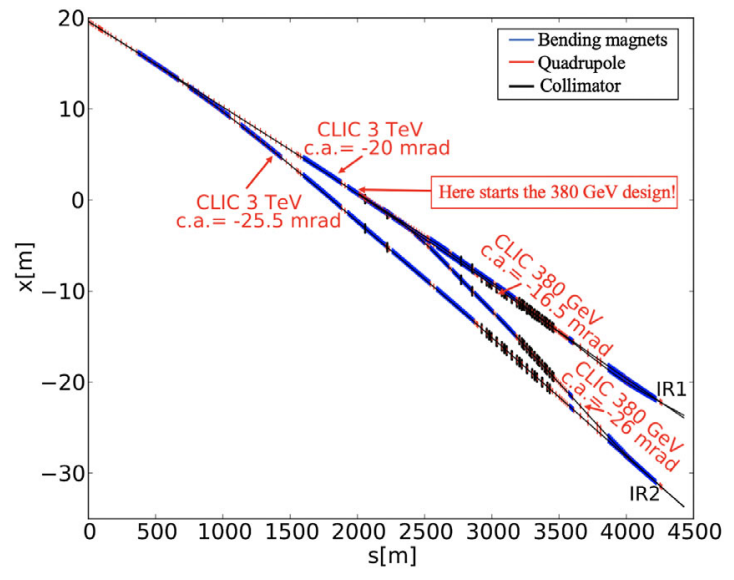

FIG. 9. Comparison between the dual BDS layout of CLIC $380 \mathrm{GeV}$ and CLIC $3 \mathrm{TeV}$.

shorter, so it goes from $2000 \mathrm{~m}$ to $4340 \mathrm{~m}$. IR1 and IR2 are fixed in their spatial locations, respectively $(4300,-21)$ and $(4340,-32)$. These results indicate the tunnel construction compatibility of the dual CLIC BDS, starting from the first energy stage of $380 \mathrm{GeV}$ and going toward higher energies also in the case of the BDS2.

Table IV summarizes the geometrical parameters and the optics functions for CLIC $3 \mathrm{TeV}$.

\section{PERFORMANCE RESULTS}

This section presents the simulations results for the dual CLIC BDS for both energy stages. In the first part the results are computed with the direct PLACET tracking procedure; in the second part the detector solenoid effects are taken into account thanks to the forward-backwardforward PLACET tracking procedure described in II A.

These simulations do not include the impedance. The study on the wakefields for the CLIC BDS can be found in [26]. In fact, also wakefield deteriorate the beam quality and in particular there are some components that can be high impedance sources in the IRs, like the resistive wall wakefields. The wakefield effect concentrates in the FFS, so a small increase of impedance is expected for the longer

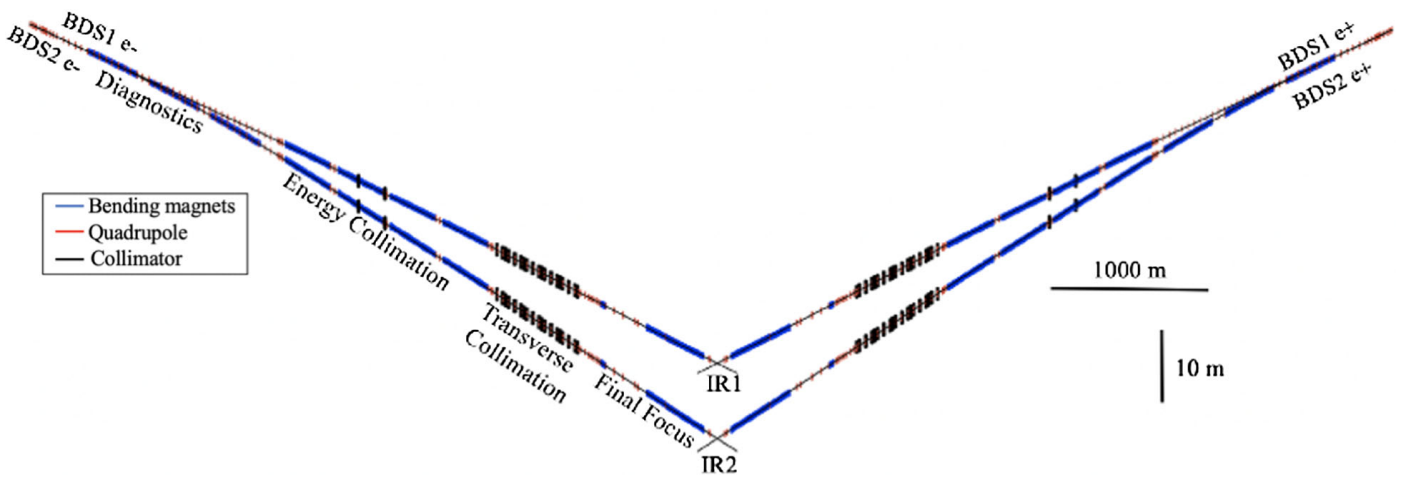

FIG. 8. Layout of the new dual CLIC 3 TeV BDS system for two IRs. 
TABLE IV. Summary table of the geometrical parameters and the optics functions for CLIC $3 \mathrm{TeV}$.

\begin{tabular}{lcccc}
\hline \hline \multicolumn{5}{c}{ CLIC 3 TeV } \\
\hline & $\begin{array}{c}\text { BDS1 } e^{+} \\
\text {(short) }\end{array}$ & $\begin{array}{c}\text { BDS1 } e^{-} \\
\text {(long) }\end{array}$ & $\begin{array}{c}\text { BDS2 } e^{-} \\
\text {(short) }\end{array}$ & $\begin{array}{c}\text { BDS2 } e^{+} \\
\text {(long) }\end{array}$ \\
\hline$\theta$ [mrad] & 0 & 0 & 2.75 & 2.75 \\
$L_{\text {dipole }[\mathrm{m}]}$ & 0 & 0 & 872.45 & 872.45 \\
$L_{\text {FODO }[\mathrm{m}]}$ & 76.72 & 76.72 & 76.72 & 76.72 \\
$L_{\text {DS }}[\mathrm{m}]$ & 1486 & 1562.75 & 1486 & 1562.75 \\
$L_{\text {BDS }}[\mathrm{m}]$ & 4190.66 & 4267.37 & 4190.66 & 4267.37 \\
c.a. $[\mathrm{mrad}]$ & 20 & 20 & 25.5 & 25.5 \\
\hline \hline
\end{tabular}

TABLE V. Beam size for the BDS1/BDS2 $e^{-}$and Luminosity simulations for the two different IRs for both CLIC energies evaluated with PLACET direct tracking procedure (no solenoid), computed for $\operatorname{BDS} 1 e^{ \pm}$and $\operatorname{BDS} 2 e^{ \pm}$.

\begin{tabular}{|c|c|c|c|c|c|c|}
\hline \multicolumn{7}{|c|}{ CLIC $380 \mathrm{GeV}$} \\
\hline & \multicolumn{2}{|c|}{$\sigma_{x}^{*}[\mathrm{~nm}]$} & \multicolumn{2}{|c|}{$\sigma_{y}^{*}[\mathrm{~nm}]$} & \multicolumn{2}{|c|}{$\mathcal{L}_{\text {TOT }}\left[10^{34} \mathrm{~cm}^{-2} \mathrm{~s}^{-1}\right]$} \\
\hline & Ideal & w/SR & Ideal & w/SR & Ideal & w/SR \\
\hline IR1 & 141 & 144 & 3.07 & 3.08 & 1.515 & 1.492 \\
\hline IR2 & 141 & 144 & 3.06 & 3.07 & 1.491 & 1.466 \\
\hline
\end{tabular}

\begin{tabular}{|c|c|c|c|c|c|c|}
\hline \multicolumn{7}{|c|}{ CLIC $3 \mathrm{TeV}$} \\
\hline & \multicolumn{2}{|c|}{$\sigma_{x}^{*}[\mathrm{~nm}]$} & \multicolumn{2}{|c|}{$\sigma_{y}^{*}[\mathrm{~nm}]$} & \multicolumn{2}{|c|}{$\mathcal{L}_{\text {TOT }}\left[10^{34} \mathrm{~cm}^{-2} \mathrm{~s}^{-1}\right]$} \\
\hline & Ideal & w/SR & Ideal & w/SR & Ideal & w/SR \\
\hline IR1 & 43.5 & 51.5 & 1.02 & 1.71 & 9.00 & 6.30 \\
\hline IR2 & 44.9 & 64.8 & 1.02 & 1.92 & 8.33 & 5.14 \\
\hline
\end{tabular}

DS but mitigations could be possible as beam-screen size increase. This needs to be studied in the future.

\section{A. Beam size and luminosity}

Table V shows the beam size evaluated with PLACET for the two different IRs. The tracking and the evaluation of the beam size has been done also in comparison with MAPCLASS [27-29] and PTC [30] codes. The results of these simulations are shown in Figs. 10 and 11, where the beam size is evaluated up to 8th order aberrations.

The simulation was done for all the different BDS, the longer and the shorter cases (considering the different longitudinal displacement of the four different new beamlines) and for both energies, $380 \mathrm{GeV}$ and $3 \mathrm{TeV}$. The first plot shows that the aberrations that mostly contribute to a beam size increase are the second order for the horizontal beam size and the third and fourth ones for the vertical beam size in the $380 \mathrm{GeV}$ case. For the $3 \mathrm{TeV}$ we have a horizontal beam size increase due to the third order aberrations while a vertical beam size increase due to fourth order aberrations. Due to these results, a further optimization of the $3 \mathrm{TeV}$ cases was needed. This was
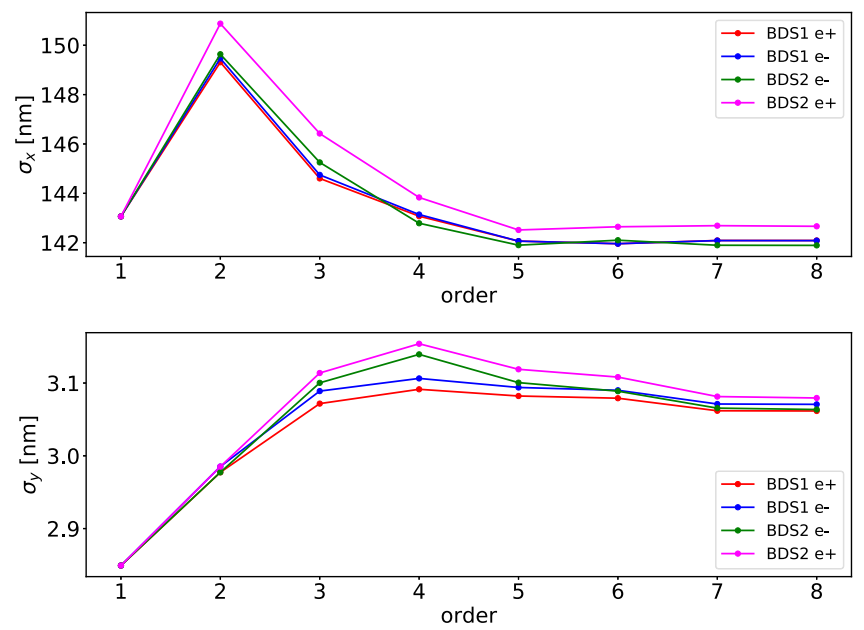

FIG. 10. $\sigma_{x}^{*}$ and $\sigma_{y}^{*}$ evaluated with MAPCLASS until the 8th order aberrations for the $380 \mathrm{GeV}$ case.
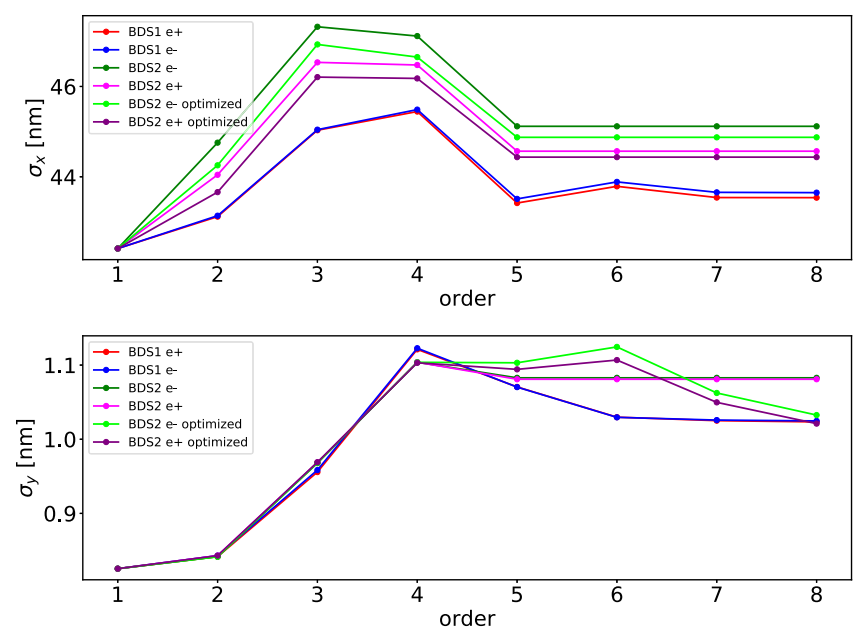

FIG. 11. $\sigma_{x}^{*}$ and $\sigma_{y}^{*}$ evaluated with MAPCLASS until the 8th order aberrations for the $3 \mathrm{TeV}$ case.

performed by correcting the aberrations that mostly increase the beam size with a pair of sextupoles in the new DS. The sextupoles were added at locations with a large dispersion and $\beta_{x}$ with a $\pi$ phase advance between them. The integrated sextupoles strength $K_{2} \mathrm{~L}$ has been set to $-0.0205 \mathrm{~m}^{-2}$ for both sextupoles, with $\mathrm{L}=0.5 \mathrm{~m}$. Thanks to that, a small improvement of the beam size trend for the IR2 can be seen in Fig. 11. Furthermore, from Table V and Figs. 10, 11 we see that the beam sizes from the different simulation codes show consistent results.

Table V shows the luminosity performance for the two IRs for both CLIC energies. Luminosities are evaluated with GUINEA-PIG using two bunches tracked along BDS1 and BDS2, respectively, with direct PLACET tracking.

\section{B. Detector solenoid effects}

The effect of the detector solenoid was taken into consideration only in the second part of the study as 


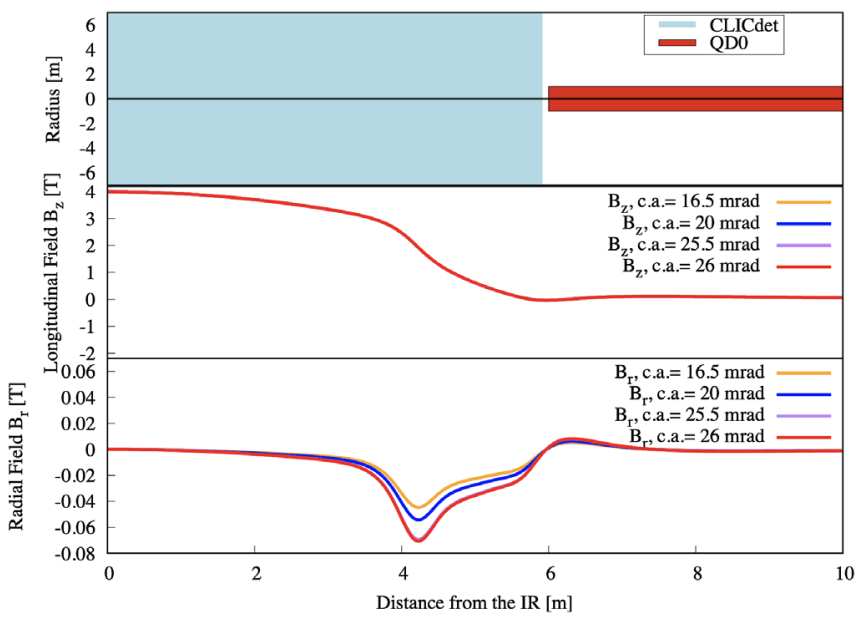

FIG. 12. The radial and the longitudinal solenoid magnetic fields along the last $10 \mathrm{~m}$ before the IR in the solenoid reference system.

described in Sec. II A. The residual transverse magnetic field from the CLICdet solenoid is considered negligible beyond $10 \mathrm{~m}$ from the IP on the beamline. Different crossing angles imply different magnetic field near the IP. In fact, the transverse solenoid magnetic field increases with the design crossing angle, as shown in Fig. 12. In particular, in Fig. 12 we can see the different radial and longitudinal fields for the different crossing angles of $16.5 \mathrm{mrad}, 20 \mathrm{mrad}, 25.5 \mathrm{mrad}$ and $26 \mathrm{mrad}$ respectively.

In Table VI we can see the results of the simulations. The solenoid effects on luminosity for CLIC $3 \mathrm{TeV}$ for the dual CLIC BDS are respectively of about $4 \%$ for IR1 and $19 \%$

TABLE VI. Luminosity performance evaluated with both PLACET tracking procedures, the direct and the forwardbackward-forward (results for ideal, with solenoid, with solenoid plus SR), for the two different IRs and for the baseline for both CLIC cases.

\begin{tabular}{|c|c|c|c|c|}
\hline \multicolumn{5}{|c|}{ CLIC $380 \mathrm{GeV}$} \\
\hline & \multicolumn{4}{|c|}{$\mathcal{L}_{\mathrm{TOT}}$} \\
\hline & \multicolumn{4}{|c|}{$\left[10^{34} \mathrm{~cm}^{-2} \mathrm{~s}^{-1}\right]$} \\
\hline & Ideal & w/sol. & w/SR & w/sol.+SR \\
\hline IR1 & 1.515 & 1.512 & 1.492 & 1.412 \\
\hline IR2 & 1.491 & 1.475 & 1.466 & 1.392 \\
\hline \multicolumn{5}{|c|}{ CLIC $3 \mathrm{TeV}$} \\
\hline & \multicolumn{4}{|c|}{$\mathcal{L}_{\mathrm{TOT}}$} \\
\hline & \multicolumn{4}{|c|}{$\left[10^{34} \mathrm{~cm}^{-2} \mathrm{~s}^{-1}\right]$} \\
\hline & Ideal & w/sol. & w/SR & w/sol.+SR \\
\hline Baseline & 9.40 & 8.65 & 6.50 & 6.22 \\
\hline IR1 & 9.00 & 8.21 & 6.30 & 6.09 \\
\hline IR2 & 8.33 & 7.59 & 5.14 & 4.17 \\
\hline
\end{tabular}

TABLE VII. Parameters of the new dual CLIC BDS System for two IRs.

\begin{tabular}{|c|c|c|c|c|}
\hline \multirow[b]{2}{*}{ CLIC } & \multicolumn{2}{|c|}{$380 \mathrm{GeV}$} & \multicolumn{2}{|c|}{$3 \mathrm{TeV}$} \\
\hline & IR1 & IR2 & IR1 & IR2 \\
\hline $\mathrm{L}^{*}[\mathrm{~m}]$ & 6 & 6 & 6 & 6 \\
\hline BDS length [m] & 2294 & 2256 & 4267 & 4191 \\
\hline Normalised emittance $\gamma \epsilon_{x}[\mathrm{~nm}]$ & 950 & 950 & 660 & 660 \\
\hline Normalised emittance $\gamma \epsilon_{y}[\mathrm{~nm}]$ & 30 & 30 & 20 & 20 \\
\hline Beta function (IP) $\beta_{x}^{*}[\mathrm{~mm}]$ & 8 & 8 & 7 & 7 \\
\hline Beta function (IP) $\beta_{y}^{*}[\mathrm{~mm}]$ & 0.1 & 0.1 & 0.12 & 0.12 \\
\hline IP beam size $\sigma_{x}^{*}[\mathrm{~nm}]$ & 144 & 144 & 51 & 65 \\
\hline IP beam size $\sigma_{y}^{*}[\mathrm{~nm}]$ & 3.08 & 3.07 & 1.7 & 1.9 \\
\hline Bunch length $\sigma_{z}[\mu \mathrm{m}]$ & 70 & 70 & 44 & 44 \\
\hline rms energy spread $\delta_{p}[\%]$ & 0.3 & 0.3 & 0.3 & 0.3 \\
\hline Bunch population $N_{e}\left[10^{9}\right]$ & 5.2 & 5.2 & 3.72 & 3.72 \\
\hline Number of bunches $n_{b}$ & 352 & 352 & 312 & 312 \\
\hline Repetition rate $f_{\text {rep }}[\mathrm{Hz}]$ & 50 & 50 & 50 & 50 \\
\hline Crossing angle [mrad] & 16.5 & 26 & 20 & 25.5 \\
\hline Luminosity $\mathcal{L}_{\mathrm{TOT}}\left[10^{34} \mathrm{~cm}^{-2} \mathrm{~s}^{-1}\right]$ & 1.41 & 1.39 & 6.09 & 4.17 \\
\hline
\end{tabular}

for IR2. For comparison, a reference for the impact of all the past experiment solenoids on the CLIC luminosity can be found in [31].

Table VII shows a summary of all the parameters of interest of the new CLIC dual BDS design taking into consideration also the solenoid effects for the luminosity evaluation.

To try to bring the current CLIC baseline $\left(\mathrm{L}^{*}=6 \mathrm{~m}\right)$ and the CLIC Dual BDS1 design to the same luminosity performance the possibility of adding an antisolenoid is explored.

A simulation with the new baseline design but with the $\mathrm{SiD}$ experiment configuration, that includes the antisolenoid, has been done. The crossing angle is $20 \mathrm{mrad}$. In Fig. 13 we can see the different radial and longitudinal fields along the beam line for the $\mathrm{SiD}$ detector respectively with (blue, solid) and without (green, dashed) the antisolenoid. This can be compared to the field of the CLICdet
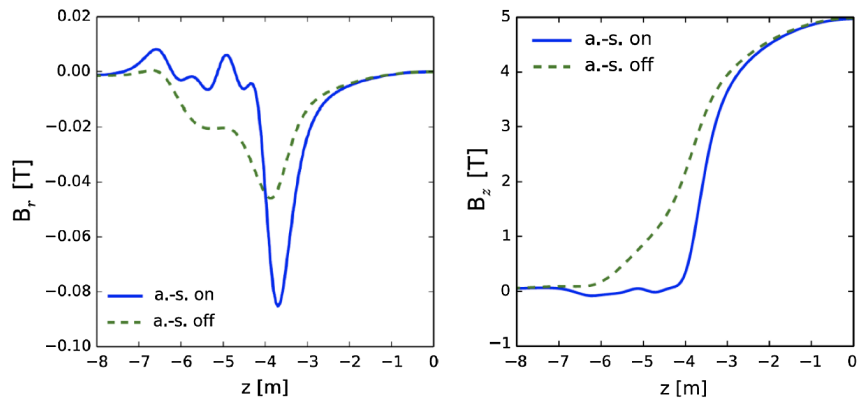

FIG. 13. The radial $\left(B_{r}\right)$ and longitudinal $\left(B_{z}\right)$ SiD solenoid field for $\mathrm{L}^{*}=3.5 \mathrm{~m}$ in [6] with (blue, solid) and without (green, dashed) antisolenoid, along a beamline with a $10 \mu \mathrm{rad}$ inclination with respect to the solenoid axis. The IP is at $0 \mathrm{~m} \mathrm{[17].}$ 
showed in Fig. 12. In the case of $\mathrm{SiD}$ experiment the central field is $5 \mathrm{~T}$ while for the CLICdet is $4 \mathrm{~T}$, so the comparison is pessimistic. In fact, a central field of $4 \mathrm{~T}$ plus an antisolenoid could improve even more the situation in our specific case. The presence of the antisolenoid leads to a decrease of the SR effects, the optical aberrations and the solenoid stray fields. So, adding an antisolenoid to the dual CLIC BDS1 could reduce luminosity loss by at least $3 \%$ from the total of $4 \%$ coming from the solenoid and the SR effects. This option could be considered in future CLIC designs.

\section{DISCUSSION AND CONCLUSIONS}

The novel dual BDS design presented in this work has the potential of making CLIC more competitive versus other future collider projects, such as the Future Circular Collider (FCC) and the Circular Electron Positron Collider (CEPC), by enabling collisions in two detectors.

This study leads to a very important result never evaluated before: the impact of the solenoid field on the CLIC baseline design with an $\mathrm{L}^{*}$ of $6 \mathrm{~m}$. In fact, the impact on the luminosity performance of CLIC $3 \mathrm{TeV}$ for the detector solenoid field is about $4 \%$ for the CLIC current baseline design and for the dual CLIC BDS1 and about $19 \%$ for the dual CLIC BDS2. This represents the total luminosity loss that cannot be corrected once the design is fixed.

Considering the detector solenoid effects there is in total a luminosity performance loss from the baseline design for the CLIC $3 \mathrm{TeV}$ of about $2 \%$ at the IR1 and about $33 \%$ of luminosity performance loss at the IR2, both with respect to the previous design but including the solenoid, $6.22 \times 10^{34} \mathrm{~cm}^{-2} \mathrm{~s}^{-1}$. The impact on CLIC $380 \mathrm{GeV}$ is still negligible for the solenoid field effects.

A summary table of all the luminosity evaluated for both CLIC energy stage scenarios and for both IRs and for the baseline can be found in Table VI.

The possibility to add the antisolenoid reduces the luminosity losses from about $4 \%$ to about $1 \%$ and leads to the same luminosity performances in the case of the baseline design and the dual CLIC BDS1 design. For the dual CLIC BDS2 instead we could not estimate the potential since the magnetic design of the solenoid does not exist for now.

To conclude, we can say that the results obtained can be considered good enough in terms of feasibility to possibly consider the CLIC dual BDS as a real candidate for the future linear collider project.

Further improvements can still be performed for the dual BDS layout in order to recover part of the luminosity performance, especially for the BDS2 of the CLIC $3 \mathrm{TeV}$ case. Currently all DS bending is placed in BDS2 however this could be distributed between BDS1 and BDS2 (with opposite angle). This would reduce luminosity loss in IR2 and increase it in IR1. Another option could be to do a longer BDS to reduce the impact of the SR in the BDS2, followed by optics improvements of the dual BDS. Furthermore, to try to reduce the impact of the solenoid effects instead for the BDS2, the antisolenoid is the best option to cancel a good fraction of luminosity losses. These could improve even more the real potential, already shown in this paper, of the CLIC dual BDS design.

\section{ACKNOWLEDGMENTS}

The authors would like to acknowledge Lucie Linssen and Konrad Elsener for the helpful discussions on the layout design of the two detectors and Roberto Corsini and Daniel Schulte for the interest and the support on the work.

[1] High-Luminosity Large Hadron Collider (HL-LHC): Technical Design Report V. 0.1, CERN Yellow Reports: Monographs (CERN, Geneva, 2017).

[2] The International Linear Collider Technical Design Report, Technical Report, 2013.

[3] P. Bambade, T. Barklow, T. Behnke, M. Berggren, J. Brau, P. Burrows, D. Denisov, A. Faus-Golfe, B. Foster, K. Fujii et al., The international linear collider: A global project, arXiv:1903.01629.

[4] A. Robson, P. Burrows, N. C. Lasheras, L. Linssen, M. Petric, D. Schulte, E. Sicking, S. Stapnes, and W. Wuensch, The compact linear e+ e- collider (clic): Accelerator and detector, arXiv:1812.07987.

[5] R. Tomás, Overview of the compact linear collider, Phys. Rev. ST Accel. Beams 13, 014801 (2010).

[6] CLIC Conceptual Design Report, Vol. 1, Tech. Report No. CERN-2012-007.

[7] F. Plassard, A. Latina, E. Marin, R. Tomás, and P. Bambade, Quadrupole free detector optics design for the compact linear collider final focus system at 3 tev, Phys. Rev. Accel. Beams 21, 011002 (2018).

[8] F. Plassard, Optics optimization of longer $\mathrm{L}^{*}$ beam delivery system designs for CLIC and tuning of the ATF2 final focus system at ultra-low $\beta^{*}$ using octupoles, Ph.D. thesis, Universit'e Paris-Saclay, 2018 [Report No. CERN-THESIS-2018-223].

[9] A. Seryi, Beam Delivery Layout For The Next Linear Collider, Technical Report (Stanford Linear Accelerator Center, Menlo Park, CA (US), 2004).

[10] G. A. Blair, Beam delivery system in ILC, Tech. Report, 2006.

[11] V. I. Telnov, The lay-out of the photon collider at the international linear collider, Pramana 69, 1177 (2007).

[12] O. R. B. Garcia, Beam dynamics in the final focus section of the future linear collider, Ph.D. thesis, Orsay, LAL, 2015 [CERN-THESIS-2015-115].

[13] M. Sands, Emittance growth from radiation fluctuations, Tech. Rep. (Stanford Linear Accelerator Center, Menlo Park, CA (USA), 1985).

[14] D. Schulte and F. Zimmermann, The crossing angle in clic, in PACS2001. Proceedings of the 2001 Particle Accelerator Conference (Cat. No. O1CH37268) (IEEE, New York, 2001), Vol. 1, pp. 148-150. 
[15] Y. Nosochkov and A. Seryi, Compensation of detector solenoid effects on the beam size in a linear collider, Phys. Rev. ST Accel. Beams 8, 021001 (2005).

[16] V. Telnov, Optimization of the beam crossing angle at the ILC for e+ e- and $\gamma \gamma$ collisions, J. Instrum. 13, P03020 (2018).

[17] Y. I. Levinsen, B. Dalena, R. Tomás, and D. Schulte, Impact of detector solenoid on the compact linear collider luminosity performance, Phys. Rev. ST Accel. Beams 17, 051002 (2014).

[18] L. Linssen, A. Miyamoto, M. Stanitzki, and H. Weerts, Physics and detectors at CLIC: CLIC conceptual design report, arXiv:1202.5940.

[19] M. Aicheler, P. Burrows, M. Draper, T. Garvey, P. Lebrun, K. Peach, N. Phinney, H. Schmickler, D. Schulte, and N. Toge, A Multi-TeV Linear Collider Based on CLIC Technology: CLIC Conceptual Design Report, CERN Yellow Reports: Monographs (CERN, Geneva, 2012).

[20] N. A. Tehrani, J. Blaising, B. Cure, D. Dannheim, F. D. Ramos, K. Elsener, A. Gaddi, H. Gerwig, S. Green, C. Grefe et al., Clicdet: The post-cdr clic detector model, Report No. CLICdp-Note-2017-001, 2017.

[21] M. Aicheler, P. Burrows, N. C. Lasheras, R. Corsini, M. Draper, J. Osborne, D. Schulte, S. Stapnes, and M. Stuart (CLIC Accelerator Collaboration), The Compact Linear Collider (CLIC)_Project Implementation Plan, CERN
Yellow Reports: Monographs (CERN, Geneva, 2019), p. 247.

[22] D. Schulte, T. D’Amico, G. Guignard, and N. Leros, CERN Report No. CERN/PS-2001-028 (AE).

[23] D. Schulte, Beam-beam simulations with GUINEA-PIG, Technical Report, 1999.

[24] H. Grote and F. Schmidt, Mad-x-an upgrade from mad8, in Proceedings of the 2003 Particle Accelerator Conference (IEEE, New York, 2003), Vol. 5, pp. 3497-3499.

[25] P. J. Bryant, Insertions, 10.5170/CERN-1994-001.159.

[26] D. Arominski, A. Latina, and D. Schulte, Resistive Wall Effects in the CLIC Beam Delivery System (JACoW Publishing, Geneva, Switzerland, 2019), paper MOPGW071, pp. 258-261, https://doi.org/10.18429/ JACoW-IPAC2019-MOPGW071.

[27] R. Tomás, MAPCLASS: a code to optimize high order aberrations, Technical Report, 2007.

[28] R. Tomás, Nonlinear optimization of beam lines, Phys. Rev. ST Accel. Beams 9, 081001 (2006).

[29] D. Martinez, A. Rosam, R. Tomás, and R. De Maria, Report No. CERN-ATS-Note-2012-087 TECH, 2012.

[30] E. Forest, F. Schmidt, and E. McIntosh, Introduction to the polymorphic tracking code, KEK report 3, 2002 (2002).

[31] B. Dalena, D. Schulte, and R. Tomas, Impact of the Experiment Solenoid on the CLIC Luminosity, Technical Report No. CERN-ATS-2010-082, 2010. 